\title{
O cuidado ao adolescente com câncer na perspectiva da equipe multiprofissional
}

\author{
Tamara Santos de Soura* \\ Letícia Macedo Gabarra**
}

\begin{abstract}
Resumo
O adoecimento por câncer na adolescência apresenta especificidades do ponto de vista biopsicossocial, o que coloca desafios à equipe na prestação de uma assistência de qualidade. Diante disso, este estudo teve como objetivo compreender a vivência do cuidado ao adolescente com câncer, na perspectiva da equipe multiprofissional de um Serviço de Onco-Hematologia adulto, de um hospital geral do sul do país, que atende adolescentes a partir dos quinze anos. Para tal, foram realizadas entrevistas semiestruturadas com nove profissionais que compõem a equipe multiprofissional. A análise qualitativa foi feita por meio da análise de conteúdo. Os dados foram organizados em quatro categorias: especificidades do cuidado ao adolescente com câncer, sentimentos mobilizados nos profissionais de saúde, estratégias de enfrentamento utilizadas pela equipe e desafios na assistência. A partir dos resultados, identificou-se que o cuidado ao adolescente com câncer tem impacto emocional significativo nos profissionais de saúde, exigindo investimento técnico e emocional para que eles possam suportar tal desafio.

Palavras-chave: câncer, adolescência, equipe de assistência ao paciente.
\end{abstract}

\section{Care for adolescents with cancer from the perspective of the multidisciplinary team}

\begin{abstract}
Teenage cancer disease presents biopsychosocial specificities, which poses challenges to the team in the provision of quality care. Therefore, this study aimed to understand the experience of care for adolescents with cancer, from the perspective of the multiprofessional team of an adult Onco-Hematology Service, of a general hospital in the south of the country, which serves adolescents from the age of fifteen. For that, semi-structured interviews were conducted with nine professionals who make up the multiprofessional team. The analysis was qualitative, through the content analysis method. The data were organized into four categories: specifics of adolescent care with cancer, feelings mobilized in health professionals, coping strategies used by the team, and challenges in care. From the results, it was identified that the care to the adolescent with cancer has significant emotional impact on the health professionals, requiring their technical and emotional investment.
\end{abstract}

Key words: cancer, adolescence, patient care team.

* Psicóloga pela UFRGS e especialista em Saúde pela UFSC.

** Psicóloga e especialista em psicologia Hospitalar pela UNESP e especialista em Saúde da Família pela UFSC. Docente da Faculdade CESUC. Mestre e Doutora pela UFSC. 


\section{Introdução}

O câncer é um problema de saúde pública que tem tido taxas de incidência aumentadas nas últimas décadas, especialmente quando se trata de crianças e adolescentes (Brasil, 2008). De acordo com os dados do Instituto Nacional do Câncer - INCA (Brasil, 2013), entre o período de 1983 a 2005, foram cadastrados 3.199 pacientes na faixa etária entre 0 e 19 anos $(2,8 \%$ do total de casos de câncer). Focando especificamente na adolescência é importante buscar recursos terapêuticos para atender a esta população e seus familiares, tendo em vista que o câncer traz à vida destes, novas experiências que são vividas de forma peculiar por cada sujeito (Volpini, 2007) e afetam toda a dinâmica familiar (Souza \& Santos, 2015).

De acordo com Presti, Macedo, Karan, Rodrigues e Petrilli (2012), o câncer na adolescência apresenta características peculiares se comparado à outras faixas etárias, motivo pelo qual esta população necessita de uma atenção especializada. As neoplasias malignas que atingem os adolescentes apresentam variações do comportamento biológico, o que impõe desafios à equipe na escolha por protocolos mais leves, utilizados para os adultos, ou mais agressivos, geralmente indicados para crianças. A Leucemia, por exemplo, está entre os tumores malignos que mais atingem os adolescentes, sendo considerada de alto risco nesta população (Petrilli \& Karan, 2008).

Em relação à classificação de adolescência, o Estatuto da Criança e do Adolescente (Brasil, 1990), considera adolescente a pessoa entre os 12 e 18 anos de idade. No entanto, o Ministério da Saúde, na formulação e execução das políticas públicas considera a faixa etária de 10 a 24 anos, com a denominação de adolescentes e jovens (Brasil, 2010).

De acordo com Aberastury, Knobel, Ferrer, Goldstein, Zarast, Kalina, Paz e Rolla (1990, p 15), “a adolescência é um momento crucial na vida do homem e constitui a etapa decisiva de um processo de desprendimento" e busca pela própria identidade. Nesse sentido, embora caracterizada como um processo de transição do ciclo vital a adolescência não deve ser vista apenas como uma passagem para a vida adulta, tendo em vista que a criança entra na adolescência com muitos conflitos e incertezas e precisa sair dela com a maturidade estabilizada, com caráter e personalidade adultos. Salienta-se que tais mudanças se dão através de um processo permeado por ambivalências, contradições e alterações de humor, características estas consideradas normais e esperadas para esta fase do desenvolvimento (Aberastury \& Knobel, 1989). Neste contexto de conflitos, incertezas e necessidade de adaptação e mudanças, a adolescência é vivida como um momento de crise, sendo que a doença potencializa as crises já esperados nesta fase do desenvolvimento, podendo ser vista como algo vergonhoso, sinal de fraqueza ou até mesmo castigo (Laterza \& Gaspar, 2016).

Diante do exposto acima, não se pode desconsiderar o impacto do adoecer no paciente e na família. No que se refere ao adolescente, além dos aspectos emocionais, o desenvolvimento físico também é alterado com o adoecimento, sendo as mudanças próprias da puberdade (menarca, aumento da estatura, etc.) muitas vezes adiadas (Laterza \& Gaspar, 2016). Além disso, muitas mudanças indesejáveis acontecem como efeito do tratamento, sendo elas: anemia, náuseas, vômitos, febre, queda de cabelo, entre outras (Brasil, 2013), o que causa preocupação significativa com a aparência física na maioria dos adolescentes, "gerando sentimentos de angústia que podem levá-los ao isolamento" (Souza \& Santos, 2015, p. 69).

Levando em consideração as mudanças físicas e/ ou emocionais Laterza e Gaspar (2016) destacam a importância do paciente se apropriar de sua doença e ter a oportunidade de conversar, tanto com os profissionais quanto com os familiares, sobre as questões que possam surgir nesse processo. A falta de atenção às reações emocionais consequentes do adoecimento "pode provocar dificuldades psicológicas e efeitos graves que dificultam a aceitação do diagnóstico, pode prejudicar o tratamento e levar, algumas vezes, até a sua interrupção" (Laterza \& Gaspar, 2016, p. 376).

Considerando a fase do desenvolvimento vivida por esses pacientes e o fato de que os pais, por vezes encontram-se fragilizados, dispondo de poucos recursos emocionais para preparar os filhos para as mudanças decorrentes do adoecimento, é necessário, por parte dos profissionais, uma sensibilidade aguçada para prestar suporte aos usuários (Fermo, Lourençatto, Medeiros, Anders \& Souza, 2014). Sendo assim, além da atenção ao paciente, a equipe multidisciplinar deve direcionar suas intervenções ao cuidador, estabelecendo uma relação de empatia com o mesmo, buscando a manutenção de sua qualidade de vida e a redução da sobrecarga de cuidado (Rubira, Marcon, Belasco, Gaíva \& Espinosa, 2012).

No estudo realizado por Fermo, Lourençatto, Medeiros, Anders e Souza (2014), com objetivo de "descrever o caminho percorrido pela criança, adolescente e suas famílias, desde o início dos sinais e sintomas até a definição do diagnóstico de câncer" (p. 54), os autores evidenciaram a fragilidade da organização dos serviços de saúde e o despreparo de muitos profissionais, tanto 
pela demora no acesso, quanto pelo não atendimento das necessidades de pacientes e familiares. Diante disso, Presti, Macedo, Karan, Rodrigues e Petrilli (2012), destacam que o acesso dos adolescentes aos serviços especializados deve ser facilitado e os serviços precisam se adaptar às necessidades específicas dessa faixa etária, de modo a oferecer o suporte e tratamento adequado. Nesse sentido, os autores destacam que as equipes precisam se aperfeiçoar, não só do ponto de vista técnico-científico, mas também no que se refere ao apoio e escuta atenta (Fermo, Lourençatto, Medeiros, Anders \& Souza, 2014).

Além das questões relacionadas à assistência ao paciente e família, o profissional se depara com suas próprias questões relativas ao adoecimento que, caso não tenham a devida atenção, podem causar prejuízos à sua qualidade de vida e saúde mental. Segundo Campos (1995), "todos os aspectos relacionados à doença, ao doente e ao adoecer estão presentes também na vida dos profissionais que atuam na área hospitalar e os inquietam" (p. 50). Nesse sentido, o cuidado ao adolescente com câncer desperta sentimento de tristeza e impotência nos profissionais (Oliveira \& Firmes, 2012), que, por vezes, se utilizam do distanciamento como estratégia para não entrar em contato com o sofrimento (Cano \& Moré, 2016; Oliveira \& Firmes, 2012).

Segundo Ortolan e Gaspar (2013), "o clima emocional da oncologia é permeado por dor e sofrimento e isso reflete na saúde mental dos profissionais" (p. 213). Diante disso, para que a assistência seja efetiva, é fundamental a habilidade do profissional em lidar com os próprios sentimentos, de forma que não influenciem na qualidade do cuidado (Oliveira \& Firmes, 2012).

Em decorrência dos eventos estressores advindos da prática profissional no contexto da oncologia, Cano e Moré (2016) realizaram um estudo que "objetivou caracterizar as estratégias de enfrentamento psicológico utilizadas por médicos oncologistas" (p.1). Na referida pesquisa, as autoras identificaram que estes profissionais se utilizam de estratégias variadas, entre elas: estratégias focalizadas no problema, na emoção e busca por suporte social . Em relação aos objetivos as estratégias focalizadas no problema buscam a modificação da realidade e do que causa estresse e ameaça; as centralizadas na emoção buscam a minimização de reações emocionais diante de situações de difícil manejo; e "a busca por suporte social, seja através de apoio instrumental, emocional ou de informações, caracteriza-se como um recurso de enfrentamento psicológico, exercendo função de apoio, suporte, no sentido de fornecer sustentação" (Cano \&
Moré, 2016, p. 6). Tendo em vista a variedade de recursos de enfrentamento utilizados pelos profissionais, as autoras ressaltam a importância de espaços de acolhimento, "tanto para trocas técnicas, quanto de sentimentos - pois, apesar do esforço em retê-los, esses acabam sendo extravasados, seja de maneira adequada ou inadequada" (Cano \& Moré, 2016, p. 7).

Diante do exposto acima, pressupõe-se que a vivência do câncer na adolescência afeta diretamente paciente e familiares, podendo, ainda, causar impacto nos profissionais, influenciando na qualidade da assistência prestada. Por entender que a vivência do câncer na adolescência pode ter repercussões distintas do que em outras fases do desenvolvimento, pressupõe-se que a equipe que vai prestar assistência a esse paciente pode se deparar com uma série de desafios e precisa, de alguma forma, estar preparada para atender às demandas apresentadas pelo paciente e sua família. Diante disso, justifica-se a importância deste estudo que tem como objetivo compreender a vivência do cuidado ao adolescente com câncer na perspectiva da equipe multiprofissional de um Serviço de Onco-Hematologia adulto em hospital geral.

\section{Método}

Os participantes deste estudo foram acessados em um serviço de onco-hematologia que atende adolescentes, mas majoritariamente adultos, localizado em um hospital geral no sul do país. No referido serviço, a equipe é composta por profissionais efetivos de diferentes categorias da área da saúde (Enfermagem, Farmácia, Fisioterapia, Medicina, Nutrição, Odontologia, Psicologia e Serviço Social, entre outros) e profissionais terceirizados que compõem a equipe de apoio (copa, recepção, segurança e serviços gerais).

Para execução desta pesquisa, foi realizado contato pessoalmente com 12 profissionais e agendada a entrevista conforme a disponibilidade dos mesmos, havendo uma negativa de participação e dois profissionais que desmarcaram a entrevista, devido à rotina de trabalho. Sendo assim, foram realizadas entrevistas semiestruturadas com nove profissionais, utilizando-se o critério de amostragem por saturação, considerando que a partir de certo número de entrevistas os dados passam a apresentar certa redundância ou repetição (Fontanella, Ricas \& Turato, 2008). Foram entrevistados profissionais das seguintes categorias: Medicina (2), Enfermagem (2), Psicologia (1), Farmácia (1), Nutrição (1), Serviço Social (1) e Equipe de apoio (Recepção) (1). Utilizou-se como critérios de inclusão a atuação direta com pacientes adolescentes com câncer 
e estar na equipe multiprofissional por no mínimo um ano. Visando preservar a identidade dos profissionais, os participantes foram numerados de P1 a P9, por ordem de realização das entrevistas, de forma que não fossem identificados por categoria profissional.

A coleta de dados ocorreu entre o período de novembro e dezembro de 2017. As entrevistas tiveram duração aproximada de trinta minutos e foram gravadas com a autorização dos participantes e transcritas na íntegra para posterior análise.

Os dados das entrevistas foram analisados de forma qualitativa através do método da análise de conteúdo, constituído pelos procedimentos metodológicos de categorização, inferência, descrição e interpretação (Minayo, 2011). Após a transcrição das entrevistas procedeu-se a uma leitura exaustiva das mesmas para posterior organização dos dados em categorias, de acordo com os objetivos do trabalho.

Salienta-se que este estudo baseou-se na resolução 466/12 do Conselho Nacional de Saúde e foi realizado de acordo princípios éticos que normatizam as pesquisas com seres humanos (Brasil, 2012), tendo sido aprovado pelo Comitê de Ética em Pesquisa com Seres Humanos (CEPSH-UFSC) através do CAAE de $\mathrm{n}^{\mathrm{o}}$ 70269817.0.0000.0121, parecer $\mathrm{n}^{\circ}$ 2.345.835, emitido em 24/10/2017. Todos os profissionais que participaram da pesquisa assinaram Termo de Consentimento Livre e Esclarecido expressando a concordância em participar do mesmo e autorizando a utilização dos dados neste estudo.

\section{Resultados e discussão}

A partir das entrevistas com os profissionais, foi possível organizar os dados em quatro categorias: (1) especificidades do cuidado ao adolescente com câncer, (2) sentimentos mobilizados nos profissionais de saúde, (3) estratégias de enfrentamento utilizadas pela equipe e (4) desafios na assistência. Para melhor organização e compreensão dos dados, optou-se por descrever as categorias separadamente, no entanto, é importante ressaltar que as mesmas se articulam.

\section{Especificidades do cuidado ao adolescente com câncer}

Nesta categoria, os participantes indicaram as especificidades no cuidado ao paciente adolescente, tendo em vista que algumas doenças se apresentam de forma mais agressiva, necessitando, consequentemente, de intervenções mais intensivas, mas que nem sempre garantem uma resposta efetiva. Segundo a literatura, as neoplasias apresentam variações de comportamento biológico nos adolescentes (Petrilli \& Karan, 2008) o que, de acordo com a fala dos profissionais (P1, P6, P7, P8 e P9) coloca a equipe constantemente em contato com a questão da imprevisibilidade e com os desafios a respeito da tomada de decisão sobre os protocolos a serem aplicados (mais leves indicados para adultos ou mais agressivos, geralmente utilizados com crianças) e, até mesmo, sobre o momento de interromper o tratamento, como pode ser visto na fala de um dos profissionais:

O tratamento desses pacientes é um grande desafio. (...) a gente tem um paciente que tem, pelos exames, todos os requisitos para que o tratamento seja bom e isso às vezes não acontece (...), é imprevisível. E frente a tudo isso, o que fazer? Por que muitas vezes a gente é levado a pensar 'ah, dobra a dose, encurta o tempo entre uma aplicação e outra', só que essas coisas, teoricamente, no papel aceitam, mas na vida real a gente pode até mesmo acelerar o processo de morte e isso pode ser até criminoso (P1).

A dificuldade na escolha dos tratamentos, ratifica a própria complexidade que nos remete a adolescência, oscilando entre o mundo infantil e o adulto. Nesse sentido, além das questões biológicas relacionadas a maior agressividade da doença e tratamento, a maioria dos profissionais (P1, P3, P4, P5, P6, P7, P8 e P9) identifica especificidades no manejo com o adolescente, tendo em vista as questões relativas à própria fase do desenvolvimento, caracterizada como uma fase de transição do ciclo vital e permeada por questões referentes ao processo de desprendimento e busca pela própria identidade (Aberastury \& Knobel, 1989; Aberastury et al, 1990). Diante disso, Fermo et al (2014) identificaram que os profissionais, por vezes, não estão preparados para atender as necessidades dos adolescentes, o que também foi apontado pelos participantes deste estudo, exemplificada na fala de P4:

A gente tem muito adulto ou idoso, né, e daí o adolescente tem um monte de comorbidades, um monte de situações ali que são específicas da faixa etária dele, que a gente não tá preparado pra cuidar. (...) é muito difícil cuidar deles porque eles são muito complexos e muito diferentes entre si. (...) e eu fico imaginando eles doentes, tentando achar a personalidade deles, tentando manter a individualidade...

\section{Sentimentos mobilizados nos profissionais de saúde}

Esta categoria evidencia que o cuidado ao paciente com câncer mobiliza uma série sentimentos nos profis- 
sionais, especialmente quando se trata de pacientes mais jovens, o que corrobora os dados apresentados na literatura (Cano \& Moré, 2016; Neme, Barbosa, Taborianski, Figueiredo, Kakuda, Júnior, Amaral \& Paiva, 2010). Os participantes deste estudo foram unânimes em afirmar que o adoecimento por câncer no paciente adolescente mobiliza mais a equipe se comparado ao paciente adulto ou idoso. Tal mobilização aparece associada, principalmente, à imprevisibilidade do tratamento e da vida, o que gera sofrimento significativo nos profissionais, resultando em sobrecarga física e emocional.

A partir das falas dos profissionais entrevistados, foi possível identificar sentimentos de angústia, tristeza, compaixão e impotência, como os mais prevalentes no cuidado ao adolescente. Segundo os profissionais (P1, P2, P3, P4 e P5), tais sentimentos estão presentes desde o diagnóstico e independente da idade, mas são de alguma forma intensificados quando se trata do paciente adolescente, tendo em vista que o adoecimento nesta faixa etária aparece como uma inversão/interrupção do ciclo vital:

É uma inversão do ciclo da vida, né. E além disso, tem uma situação que nesses pacientes jovens, aquela perspectiva de que ele iria viver até os $50,60,70,80 \ldots$ ele vai constituir família, ele vai ter uma série de vivências... aquilo é interrompido. É diferente de quando a gente tem um paciente de 89 anos, com 79 anos, com... enfim, 90 anos... que é um paciente que já teve uma vida, que já construiu uma série de coisas, então a gente até entende melhor essa situação (P1).

Em um estudo sobre o contato dos profissionais com a morte de pacientes oncológicos, Neme et al (2010) apontam que os profissionais, principalmente os que têm filhos, acabam por se identificar com o sofrimento de pacientes e familiares, sendo esta identificação também reconhecida por seis dos profissionais entrevistados neste estudo (P1, P3, P5, P6, P7 e P8): "no começo a angústia era porque en tinha o men filho adolescente e en vi muitos da idade dele partir, então aquele sentimento de mãe, né: ah se fosse o meu filho..." (P3).

Quando solicitado que os profissionais relatassem um caso que mais marcou sua trajetória com pacientes adolescentes, sete dos nove entrevistados (P1, P3, P5, P6, P7, P8 e P9) lembraram de casos que acabaram tendo um desfecho de óbito, sugerindo que as situações de terminalidade são as que mais mobilizam a equipe (sentimento de tristeza, frustração e impotência): “se far todo um ajuste pensando no bem do paciente, na segurança do paciente, mas por questões que fogem às minhas capacidades (...) ele não responde adequadamente e o paciente vem a ter o desfecho que a gente não quer" (P1). Nesse sentido, Neme et al (2010) destacam a necessidade de apoio aos profissionais, tendo em vista as dificuldades vivenciadas pelos mesmos, especialmente diante do contato com a morte do paciente oncológico.

Para finalizar a descrição dos dados referentes a esta categoria, é importante ressaltar que os profissionais (P1, P3, P5, P6, P7, P8 e P9) também vivenciam sentimentos de competência e gratificação pelo cuidado oferecido e pelo vínculo com pacientes e familiares: "claro que isso é uma situação que te envolve muito... pessoalmente, assim, mas, ao mesmo tempo, é muito gratificante, eu penso" (P6). Este dado corrobora os resultados apontados por Neme et al (2010), os quais identificaram que, apesar tristeza, desalento, desânimo e frustração associados ao contato com a morte de pacientes oncológicos, "envolver-se com esses pacientes também significa recompensas, significando dar muito e receber muito de volta" (p. 252).

\section{Estratégias de enfrentamento utilizadas pela equipe}

Quando questionados sobre as estratégias utilizadas para lidar com as dificuldades e com os sentimentos despertados a partir do cuidado ao adolescente com câncer, os profissionais citaram estratégias de enfrentamento focalizadas no problema, na emoção e na busca por suporte social. Tais estratégias são semelhantes às identificadas no estudo de Cano e Moré (2016) sobre as estratégias de enfrentamento de médicos oncologistas.

Entre as estratégias focalizadas no problema, que tem como objetivo a modificação do que causa estresse ou ameaça (Cano \& Moré, 2016), destaca-se a demanda dos participantes (P1, P4, P5, P6, P7 e P8) por capacitações e promoção de espaços de reflexão e troca entre os profissionais, conforme ilustrado na fala de P1:

Se a gente tivesse um acompanhamento ou uma... eu não diria supervisão... O melhor seria um acompanhamento por um profissional da área da Psicologia que não tivesse diretamente vinculado com a gente, por que seria uma amostra viciada (...) para saber como é que a pessoa está lidando com as coisas, como é que tá indo todo o andamento do serviço, até que ponto a mobilização que se teve... (...) como que a gente tá lidando com isso aí, numa situação em que a gente tem pacientes que talvez não vão ter uma cura, que o tratamento não vai ser positivo, né. 
Assim como no estudo de Cano e Moré (2016), três dos participantes desta pesquisa (P2, P3 e P4) utilizam estratégias de enfrentamento focalizadas na emoção, entre elas: distanciamento emocional, descontar o estresse e a sobrecarga em outras pessoas da equipe e distração como forma de evitar o contato com assuntos que possam mobilizar sofrimento. É importante ressaltar que, apesar de ter como objetivo a minimização das reações emocionais, as estratégias de enfrentamento focalizadas na emoção são consideradas pouco funcionais, por apresentarem efeitos momentâneos, estando associadas a uma maior incidência de síndromes relacionadas ao estresse, como a Síndrome de Burnout (Blandin \& Araujo, 2005). A fala de P6 ilustra o distanciamento de alguns profissionais em relação às questões emocionais:

"Eu percebo que quando a gente fala da técnica, ah pode conversar ali, sei lá, uma hora, até mais, a manhã inteira. Agora quando tu fala de sentimento, o que tu sente em relação àquilo, não! Pra quê?! Perda de tempo".

Além das estratégias focalizadas no problema e na emoção, a busca por suporte social (apoio instrumental, emocional ou de informações) também foi apontada pelos entrevistados (P1, P5, P6 e P8) como estratégia de enfrentamento. Nesse sentido, o compartilhamento de experiências e emoções foi apontado por alguns dos participantes (P1, P2, P4, P5, P6 e P7) como importante estratégia de enfrentamento e estes, inclusive, ressaltaram a necessidade de promoção de espaços de troca e reflexão como possibilidade de fortalecimento da equipe: "Se a gente não aproveitar a equipe pra se autocuidar (...) porque th precisa receber, metabolizar esse sentimento e ai botar pra fora. Ai tu consegue tratar. Se tu fica na superficialidade ou na defensiva... eu acho que não trata direito" (P6).

Segundo Neme et al (2010), a identificação e expressão dos sentimentos e emoções podem ser vistas como uma possibilidade de cuidado ao profissional. Nesse contexto, as reuniões multidisciplinares podem se constituir como um espaço de cuidado da equipe, através do acolhimento do sofrimento e elaboração da dor, estimulando a comunicação entre os profissionais e, inclusive, minimizando as fantasias de poderes ilimitados de cura e manutenção da vida (Ortolan \& Gaspar, 2013).

\section{Desafios na assistência}

A partir das entrevistas com os profissionais, foi possível identificar alguns desafios da assistência ao adolescente com câncer em um serviço que atende majoritariamente adultos, tendo em vista as especificidades da adolescência. Entre os desafios destacados pelos profissionais que participaram da pesquisa, destaca-se: articulação da equipe multiprofissional; estrutura física mais adequada; necessidade de atividades voltadas para os adolescentes; e promoção de espaços de escuta para os adolescentes.

Em relação à formação da equipe, a maioria dos profissionais entrevistados (P1, P2, P3, P6e P7) julga que a mesma é relativamente completa, contando com profissionais de diversas áreas. No entanto, os profissionais entendem que a articulação desta equipe se constitui como um importante desafio para que a assistência seja adequada e efetiva (P4, P5, P6 e P7).

Salienta-se que o serviço de oncologia pesquisado conta com uma reunião semanal de duas horas com a presença de toda equipe multiprofissional para discussão e tomada de decisão sobre os casos dos pacientes internados. Neste estudo, uma parcela significativa dos profissionais entrevistados (P1, P4, P5, P6, P7, P8) apontou a reunião como uma conquista importante, que os diferencia de outras equipes da mesma instituição. Entre as limitações destacadas pelos profissionais, P5 e P6 apontam que a referida reunião ainda se caracteriza como um espaço centrado nas questões médicas e que poderia ser melhor aproveitado em termos de troca entre os profissionais das diferentes áreas. Além disso, foi destacada a importância de que a reunião não seja o único espaço de comunicação entre os profissionais e que as trocas aconteçam no dia a dia (P3, P4 e P6).

Quanto à estrutura física do Serviço de Oncologia em questão, cinco dos entrevistados (P1, P2, P5, P7 e P8) entendem que a mesma não é adequada para os pacientes oncológicos em geral, tendo em vista que se trata de uma unidade de internação mista, com um percentual de leitos destinados aos pacientes oncológicos. Sendo assim, os profissionais identificam a necessidade de uma unidade exclusiva para pacientes oncológicos e um dos profissionais aponta que, tendo em vista as especificidades da adolescência, o ideal seria ter um espaço físico destinado especialmente para esta faixa etária:

Até uma idade, até 14 , às vezes até 15 , tá na Pediatria. Aí passou disso vai pro adulto. E aí deveria ter uma unidade pra essa outra faixa etária, né. (...) Eu sinto falta de uma estrutura mais adequada aqui. Isso me incomoda por causa deles. Eu acho que eles ficariam mais... se sentiriam melhor se tivesse um espaço mais adequado pra eles. Por que assim, a gente recebe muitos pacientes idosos aqui, muitos idosos (P5). 
Os dados acima corroboram com os apresentados por Souza e Santos (2015), os quais destacam a necessidade de ações voltadas para os adolescentes e adequação do ambiente hospitalar, tendo em vista as peculiaridades do adoecimento por câncer nessa faixa etária. Segundo as autoras, o fato de que os adolescentes geralmente são tratados em unidades de pediatria ou de adultos não permite a interação e troca de experiências com pessoas da mesma faixa etária.

Tendo em vista a inexistência de uma estrutura física voltada para os adolescentes no serviço pesquisado e, considerando o tempo de internação dos pacientes, foi destacado pelos profissionais (P5, P7 e P8) a necessidade de realização de atividades lúdicas e educativas voltadas para os adolescentes, no entanto, a equipe não conta com um profissional que possa assumir tal demanda.

Outro desafio levantado pelos participantes deste estudo (P1, P3, P4, P6, P7 e P9), é a promoção de espaços de escuta para os adolescentes, para que estes possam ter compreensão da sua condição clínica e possibilidade de opinar na tomada de decisões sobre as condutas do seu tratamento, através do esclarecimento de dúvidas e expressão de medos, angústias, etc. Nesse sentido, por vezes a omissão da informação ocorre na tentativa de proteger o adolescente do sofrimento, porém acaba por impedi-lo de se apropriar de sua doença (Souza e Santos, 2015), conforme explicita P6: "Talvez a diferença no adolescente seja o não ter o empoderamento sobre o que é seu: 'a minha vida, o que eu vou fazer dela, o que eu eston fazendo aqui'. Às vezes a gente tem muita influência da família e nem sempre ele se apodera disso".

$\mathrm{O}$ adoecimento na adolescência pode implicar mudanças na rotina (deixar de frequentar a escola, o trabalho e sair com os amigos), assim como mudanças na relação pais-filhos, através de uma postura de superproteção (Souza \& Santos, 2015). Salienta-se que, além da família, a própria equipe por vezes assume tal postura que pode, inclusive, representar prejuízos ao desenvolvimento dos adolescentes, conforme apontado por P9:

A estratégia é sempre dar espaço, ouvir esse adolescente, ver como tá sendo pra ele, ver... ficar muito atento pra não ter muitos ganhos secundários, porque às vezes a família... e às vezes a equipe também, na tentativa de cuidar mais... então é como se você de alguma forma pudesse promover ganhos secundários que possam estacionar ali no desenvolvimento. É como se fosse confortável demais pra ele ficar nesse papel assim. Então eu... eu acho que assim... poder ficar atento de como a equipe está lidando, pra que nesses sentimentos não se perca... ou atrapalhe esse processo.

\section{Considerações finais}

A análise dos resultados obtidos neste estudo e a revisão da literatura deixaram evidente que o câncer na adolescência apresenta especificidades do ponto de vista biológico e psicossocial, com impacto emocional significativo em todos os envolvidos, inclusive entre os profissionais. Tendo em vista tais especificidades e o fato de que os adolescentes geralmente são tratados em unidades de pediatria ou de adultos, os profissionais se deparam com uma série de desafios e muitas vezes sentem-se despreparados para lidar com as demandas da adolescência associadas à vivência do câncer. Diante disso, este estudo buscou, através da compreensão da vivência dos profissionais, possibilitar reflexões que possam, de alguma forma, contribuir para o fortalecimento das equipes e, consequentemente, para uma assistência mais qualificada e efetiva.

A partir das entrevistas, foi possível constatar que o cuidado ao adolescente oncológico mobiliza sentimentos, com os quais os profissionais nem sempre estão preparados para lidar, utilizando-se, por vezes, de estratégias de enfrentamento pouco funcionais, como o distanciamento emocional e descontar o estresse e a sobrecarga nos colegas, por exemplo. Nesse sentido, cuidar do paciente com câncer, especialmente do paciente adolescente, exige não somente investimento técnico, como também emocional. Diante disso, percebe-se a importância de promoção de espaços de reflexão e troca entre os profissionais, pois, a partir do reconhecimento dos sentimentos envolvidos a equipe passa a se autocuidar e se fortalecer, para que tais sentimentos não interfiram em sua saúde mental e na prestação do cuidado.

Com bases nos resultados apresentados, entende-se que o estudo respondeu aos objetivos propostos, possibilitando, através da compreensão da vivência dos profissionais, reflexões sobre possíveis estratégias para uma assistência mais qualificada e efetiva, podendo, ainda, subsidiar a formação profissional e o planejamento das intervenções.

Como possíveis limitações deste estudo, destaca-se que não foi possível analisar como os adolescentes e seus familiares vivenciam o adoecimento e como percebem a assistência prestada pela equipe multiprofissional. Sendo assim, sugere-se a realização de estudos com os adolescentes e familiares, para que possam ser identificadas suas necessidades, bem como a percepção dos mesmos sobre o cuidado recebido. 


\section{Referências}

Aberastury, A., Knobel, M., Ferrer, E. S. L., Goldstein, R. Z., Jarast, S. G., Kalina, E., Paz, L. R., \& Rolla, E. H. (1990). Adolescência. Porto Alegre: Artes Médicas.

Aberastury, A; Knobel, M. (1989). Adolescência normal: um enfoque psicanalítico. Porto Alegre: Artes Médicas.

Blandin, J., Araujo, D. M. (2005). Estrés laboral y mecanismos de afrontamiento: Su relación en la aparición del Síndrome de Burnout en médicos residentes del Hospital Militar "Dr. Carlos Arvelo". Archivos Venezolanos de Psiquiatria y Neurologia, 51(104), 12-15.

Brasil. Ministério da Saúde. Conselho Nacional de Saúde. (2012). Resolução $\mathrm{n}^{\circ}$ 466, de 12 de dezembro de 2012: sobre pesquisa envolvendo seres humanos. Brasília, DF. Disponível em http://bvsms.saude.gov.br/bvs/ saudelegis/cns/2013/res0466_12_12_2012.html

Brasil. Ministério da Saúde. Instituto Nacional de Câncer (2008). Câncer da criança e adolescente no Brasil: dados dos registros de base populacional e de mortalidade. Rio de Janeiro, RJ: INCA. Disponível em http://www1. inca.gov.br/tumores_infantis/pdf/livro_tumores_infantis_0904.pdf

Brasil. Ministério da Saúde. Instituto Nacional de Câncer (2013). Diagnóstico precoce do câncer na criança e no adolescente. Rio de Janeiro, RJ: INCA. Disponível em http://institutoronald.org.br/wp-content/uploads/2015/07/ diagnostico_precoce_cancer_crianca.pdf

Brasil. Ministério da Saúde. Secretaria de Atenção à Saúde. (2010). Diretrizes Nacionais para a Atenção Integral à Saúde de Adolescentes e Jovens na Promoção, Proteção e Recuperação da Saúde. Brasília, DF. Disponível em http://bvsms.saude.gov.br/bvs/publicacoes/diretrizes_nacionais_atencao_saude_adolescentes_jovens_promocao_saudepdf

Brasil. Presidência da República. Casa Civil, Subchefia para Assuntos Jurídicos (1990). Estatuto da criança e do adolescente: Lei federal no 8069, de 13 de julho de 1990. Disponível em http://www.planalto.gov.br/Ccivil_03/ leis/L8069.htm

Cano, D. S.; Moré, C. L. O. O. (2016, Jul-Set). Estratégias de Enfrentamento Psicológico de Médicos Oncologistas Clínicos. Psicologia: Teoria e Pesquisa. Brasília, v. 32, n.3, pp. 1-10. Disponível em http://www.scielo.br/ scielo.php?pid=S0102-37722016000300210\&script $=$ sci_abstract\&tlng $=$ pt

Fermo, V. C.; Lourençatto, G. N.; Medeiros, T. S.; Anders, J. C.; Souza, A. I. J. (2014, Jan-Mar) O diagnóstico precoce do câncer infanto-juvenil: o caminho percorrido pelas famílias. Esc. Anna Nery, Rio de Janeiro, v. 18, n. 1, pp. 54-59. Disponível em http://www.scielo.br/scielo.php?script=sci_arttext \&pid=S1414-81452014000100054

Fontanella, B. J. B.; Ricas, J.; Turato, E. R. (2008). Amostragem por saturação em pesquisas qualitativas em saúde: contribuições teóricas. Cad. Saúde Pública, Rio de Janeiro, 24(1):17-27. Disponível em http://www.scielo.br/ $\mathrm{pdf} / \mathrm{csp} / \mathrm{v} 24 \mathrm{n} 1 / 02 . \mathrm{pdf}$
Laterza, I. D. O.; Gaspar, K. C. (2016). O câncer na adolescência e os aspectos psíquicos relacionados à amputação. In: Angerami, V. A.; Gaspar, K. C. (Orgs). O câncer diante da Psicologia: uma visão interdisciplinar (pp. 375-390). São Paulo, SP: Casa do Psicólogo.

Minayo, M. C. S. (2011). Pesquisa social: teoria, método e criatividade. Petrópolis, RJ: Vozes. 2011.

Neme, C. M. B.; Barbosa, C. G.; Taborianski, D.; Figueiredo, P. C.; Kakuda, R. M.; Júnior, S. L. R.; Amaral, C. B. A.; Paiva, M. M. (2010). O contato com a morte de pacientes no serviço de oncologia hospitalar. In: Neme, C. M. B. N. (org.). In Psico-Oncologia: Caminhos e Perspectivas. São Paulo, SP: Summus.

Oliveira, M. C. L.; Firmes, M. P. R. (2012, Jan-Mar). Sentimentos dos profissionais de enfermagem em relação ao paciente oncológico. Rev. Min. Enferm.; 16 (1), pp. 91-97. Disponível em http://bases.bireme.br/cgi-bin/wxislind. exe/iah/online/?IsisScript $=$ iah/iah.xis\&src=google $\&$ base $=$ BDENF\&lang $=$ p\&nextAction $=\operatorname{lnk} \&$ exprSearch $=22562 \&$ indexSearch $=$ ID

Ortolan, P. E.; Gaspar, K. C. (2013). A importância do cuidado em oncologia: considerações acerca dos profissionais de saúde. In: Angerami-Camon, V. A.; Gaspar, K. C. Psicologia e Câncer (pp. 207-243). São Paulo, SP: Casa do Psicólogo.

Petrilli, A. S.; Karan, E. M. M. (2008). Oncologia e Adolescência. In: Vitalle, M.S. S.; Medeiros, E. H. G. R. (Orgs). Guia de adolescência: uma abordagem ambulatorial (pp. 371-376). Barueri, SP: Manole.

Presti, P. F.; Macedo, C. R. D.; Karan, E. M.; Rodrigues, A. H. D.; Petrilli, A. S. (2012). Estudo epidemiológico de câncer na adolescência em centro de referência. Revista Paulista de Pediatria. São Paulo, v. 30, n. 2, pp. 210-216. Disponível em http://www.scielo.br/scielo.php?pid=S0103$-05822012000200009 \&$ script $=$ sci_abstract\&tlng=pt

Rubira, E. A. Marcon, S. R.; Belasco, A. G. S.; Gaíva, M. A. M.; Espinosa, M; M. (2012). Sobrecarga e qualidade de vida de cuidadores de crianças e adolescentes com câncer em tratamento quimioterápico. Acta. Paul. Enferm., São Paulo, v. 25, n. 4, pp. 567 - 573. Disponível em http://www.scielo.br/ scielo.php?script $=$ sci_arttext\&pid $=$ S0103-21002012000400014

Souza, D. S. M.; Santos, L. G. (2015). O adolescente com câncer: o adoecimento potencializando os conflitos da adolescência. Rev. Cientifico. Fortaleza, v. 15, n. 30, pp. 61-78. Disponível em https://revistacientefico.devrybrasil. edu.br/cientefico/article/view/108

Volpini, F. S. O adolescente frente ao câncer: hospitalização e processos psicológicos. Porto Alegre: UFRGS, 2007. Disponível em https://lume.ufrgs. br/bitstream/handle/10183/12300/000605100.pdf

Submetido em: 21-2-2018

Aceito em: 18-7-2019 